Aneurysi of the ANterior Cerebral Artery

\title{
A CASE OF ANEURYSM OF THE ANTERIOR CEREBRAL ARTERY CAUSING COMPRESSION OF THE OPTIC NERVES AND CHIASMA
}

BY

\author{
S. TAYLOR HARRIS \\ LONDON
}

\begin{abstract}
ANELRISMs are among the rarer causes of compression of the optic nerves and chiasma, and although a case was described in this journal by Conway in $1926^{(3)}$, it is of interest to add another, recently observed, to the scanty literature on the subject.

As long ago as 1833 Stilling $^{(9)}$ described the case of a woman, aged 42 years, who suddenly fell unconscious in the street. She regained consciousness one hour later and was then blind. It is recorded that her pupils were dilated and scarcely reacted to light. She died twenty-four hours later, and at the autopsy a tumour of the size of a walnut was found on the base of the brain, indenting the floor of the third ventricle and the tuber cinereum, and compressing the chiasma. It took origin from the right carotid artery. In this case, as in many of those since recorded, the visual symptoms were only terminal; in fact, frequently, as Beadles ${ }^{(1)}$ has remarked, aneurysms in this position have produced no visual symptoms although they have compressed the optic nerves or the chiasma.

Hutchinson ${ }^{(5)}$ also recorded a case in which sudden loss of sight developed only a short time before death. Here the aneurysm grew from the basilar artery, and extending forwards through the interpeduncular space, compressed the chiasma and the optic nerves.
\end{abstract}

Those cases in which the aneurysm caused visual symptoms for a long period before death are of more interest to the ophthalmologist.

Byrom Bramwell(2) records the case of a man, aged 31 years, who had been an inmate of an asylum for two years. At the age of 16 years he fell from a horse and had been mentally dull since then. Vision had begun to fail about two years before he came under observation. Then Sir George Berry observed double optic atrophy, considerable reduction in central vision and bitemporal hemianopia, which was complete in the left eye and partial in the right eve. After this vision improved considerably, but again deteriorated so that when he was finally examined he had a complete bitemporal hemianopia and some loss in the left nasal field. An aneurysm the size of a small orange was found attached to the right internal carotid artery, which had eroded the base of the skull to the size of an egg in the region of the sella Turcica. The 
left optic nerve was stretched over the aneurysm as a flattened band.

Weir Mitchell(7) records a case of a man, aged 43 years, in whom vision of the left eye had been failing gradually for two and a half years and that of the right eye for two years. Both discs were atrophic, and the temporal sides of each visual field were lost. A large aneurysm was found embedded in the sella Turcica, which had been eroded. The chiasma had disappeared and no evidence that it had been ruptured could be found. It was assumed that the aneurysm grew from an abnormal artery connecting the two internal carotids below the chiasma.

Kummell's case ${ }^{(6)}$ was one of a man, aged $3 \pi$ years, whose vision had failed from each temporal side for the past three or four years. For a year the right eye had been blind, and in the left a little vision remained only in the lower nasal quadrant. He had had repeated apoplectic attacks, but no signs of involvement of the nervous system were present. A radiographic examination showed flattening of the sella Turcica. At the autopsy an aneurysm the size of a plum was found on the base of the brain. It consisted of two parts, the anterior of which had eroded the floor of the anterior fossa of the skull, and a posterior part, as large as a walnut, which compressed the chiasma and the optic nerves. The aneurysm had eroded the sphenoid, extended into the sphenoidal sinus, and was separated from the naso-pharynx by mucous mem.brane only. The pituitary body could not be discovered, and the exact origin of the aneurysm could not be determined.

Freund ${ }^{(4)}$ describes the case of a man, aged 70 years, who had had tabes dorsalis for several years. Eight years before he came under observation right-sided homonymous hemianopia had gradually developed. He had not complained of headaches nor of noises in his head. At the autopsy a spindle-shaped aneurysm, the size of a large hazel nut, which grew from the left internal carotid artery, was found compressing the optic tract.

The history of my case was as follows :

M.P., aged 46 years, a married woman, was admitted to Charing Cross Hospital, under the care of Dr. Gordon Holmes, on November 11, 1926.

She had always been delicate, but had had no illness of consequence, except an attack which she called a "nervous breakdown" seven years ago. This set in suddenly with double vision while she was travelling by train; she actually stated she saw six or seven images of every object at which she looked. The double vision lasted several months, and for a period of five months at least she was so ill that she attended a hospital, but she cannot remember 
what her symptoms then were, except that she had headaches and that she was very depressed.

In April, 1926, she noticed that her vision was defective, and about the same time she began to suffer with severe headaches. Vision deteriorated rapidly, so that in June, when she was seen by $\mathrm{Mr}$. E. Brewerton at the Royal Westminster Ophthalmic Hospital, she could read $6 / 60$ only with the left eye, and the vision of her right eye was even less. Her fundi were normal and her discs were of good colour, her right visual field was contracted concentrically to about $30^{\circ}$, and there was a large ciefect in the nasal part of the left field, especially in the upper quadrant; some loss of colour vision was also noted. She was instructed to attend the ophthalmic hospital again that her fields of vision might be more accurately charted, but she failed to do so. From this time she attended the out-patient department of charing Cross Hospital, complaining mainly of intense headaches. Her vision continued to deteriorate, and early in August she became quite blind; a few weeks later her sight returned, but she then found she was unable to see objects to her right side. In November the headaches became more severe, and were then chiefly in the left frontal region. At the same time she became drowsy and could be roused only with difficulty.

On admission to hospital she was a small, thin, pale, and illlooking woman. She still suffered with headaches, especially in the left frontal region. She was drowsy, but her mental state was otherwise normal. Her heart was normal and her pulse regular and soft, but often slow, sometimes falling to under 60 per minute; the walls of the radial vessels were not thickened. The urine contained no abnormal constituent. The Wassermann reaction was negative in both her blood and cerebro-spinal fluid, and the latter contained no excess of cells or globulin.

There was no disturbance of speech and her memory was good. The ocular movements were normal and her pupils contracted on convergence, but the left pupil did not react well- to light. The functions of her other cranial nerves were unaffected, and there was no paresis, alteration of tone, ataxia or involuntary movements in her limbs. Sensibility to tactile, painful and thermal stimuli was normal, and all her reflexes were present and unaltered, the plantars being flexor in type.

The vision of her left eye was very defective, in fact she could recognize large moving objects only in a small portion of the temporal field; central vision was lost. Unfortunately, the acuity of central vision in the right eye was not measured accurately, but she was able to recognize persons and objects in the ward and to read medium-sized print. In this eye the nasal field was almost full, but there was a complete temporal hemianopia, the blindness 
extending almost up to the fixation point. The chart (Fig. 1) represents the field of the right eye as taken with a $10 \mathrm{~mm}$. object on a perimeter at a distance of $30 \mathrm{cms}$.; the left field is only an estimation from tests by rough methods as she was not able to see the fixation point.

Both discs were now very pale, more particularly the left; the central pits were deep and shelving, and there was some pigmentary disturbance on the inner border of the right disc. The retinal arteries and veins were normal in size.

A radiographic examination of her head revealed no change in the sella Turcica and no shadows in its neighbourhood.

While in hospital she complained of very intense headache, which she referred to the left side of her forehead and her left eye. She was also troubled with nausea, but did not vomit. No other change in her state occurred till December 16, when she suddenly sat up in bed, cried out that she had become blind, and immediately fell back unconscious. Her pupils were at first small, but after about eight hours they began to dilate, the right becoming fully dilated. A lumbar puncture at this time provided fluid strongly mixed with blood. She died without regaining consciousness about twenty hours after the attack.

Autopsy. - The whole surface of the brain was covered with blood. Part of it lay in the subarachnoid space, but some was over the arachnoid and was easily washed off. The third and fourth ventricles also contained blood. On raising the brain from the base of the skull a large tense swelling was found between the inner aspects of the two temporal lobes and in front of the pons. The optic nerves were stretched over its anterior and inferior surfaces and displaced to the left. The right nerve was flattened into a band; the left also was compressed and oval on section. By cutting round the dural roof of the sella Turcica the pituitary gland was removed intact with the infundibulum, which projected up from the left side of the tumour. As seen from the inferior surface, the tumour was spherical and measured from $2.5 \mathrm{cms}$. by $3 \mathrm{cms}$. across.

The brain was divided by mesial sagittal section (Fig. 2) through brain-stem and the corpus callosum; the section divided the tumour approximately in half, though it projected farther into the angle between the inner aspect of the temporal and frontal lobes on the right side than on the left. It was filled with firmly clotted blood, the removal of which revealed a glistening surface resembling that of the inner side of a large artery, with some small patches of atheromatous-like thickening. There was a large rent on the posterior surface of the tumour where it lay against the anterior surface of the pons. This was the site of rupture. At about the middle of the left lateral aspect of the tumour there was a small 

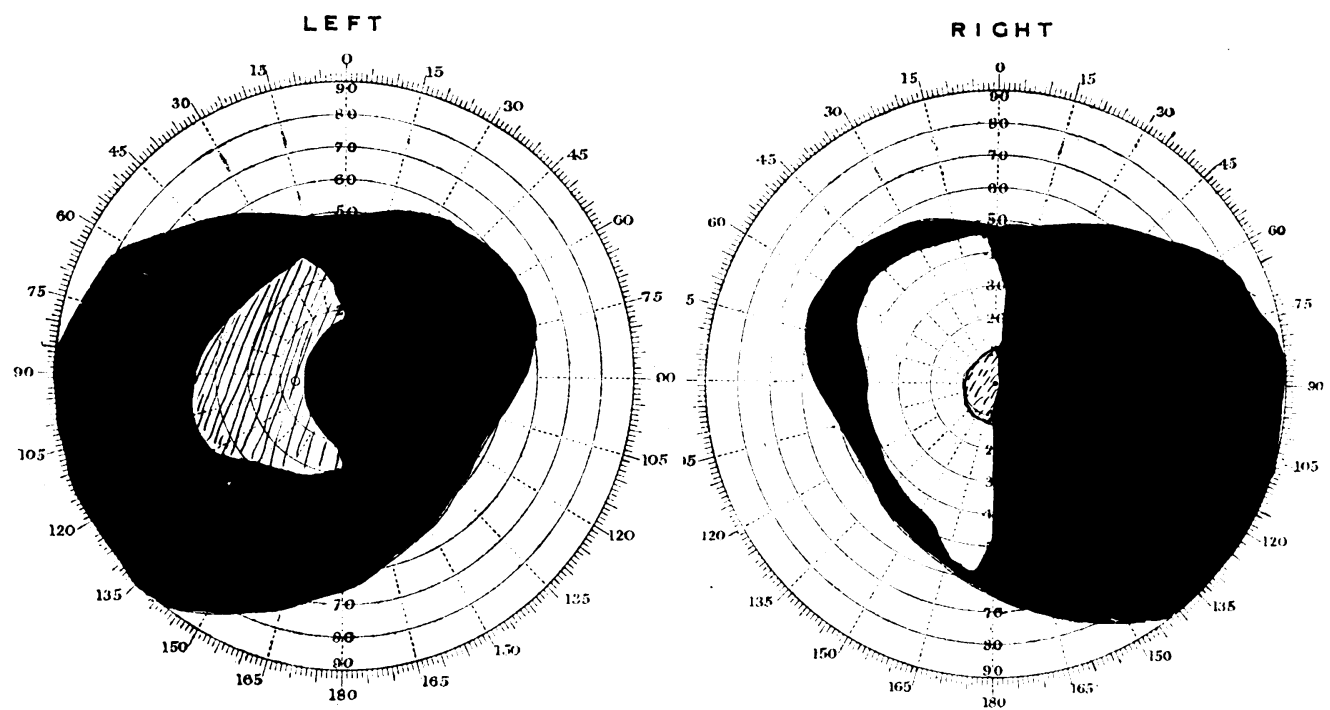

Fici, 1.

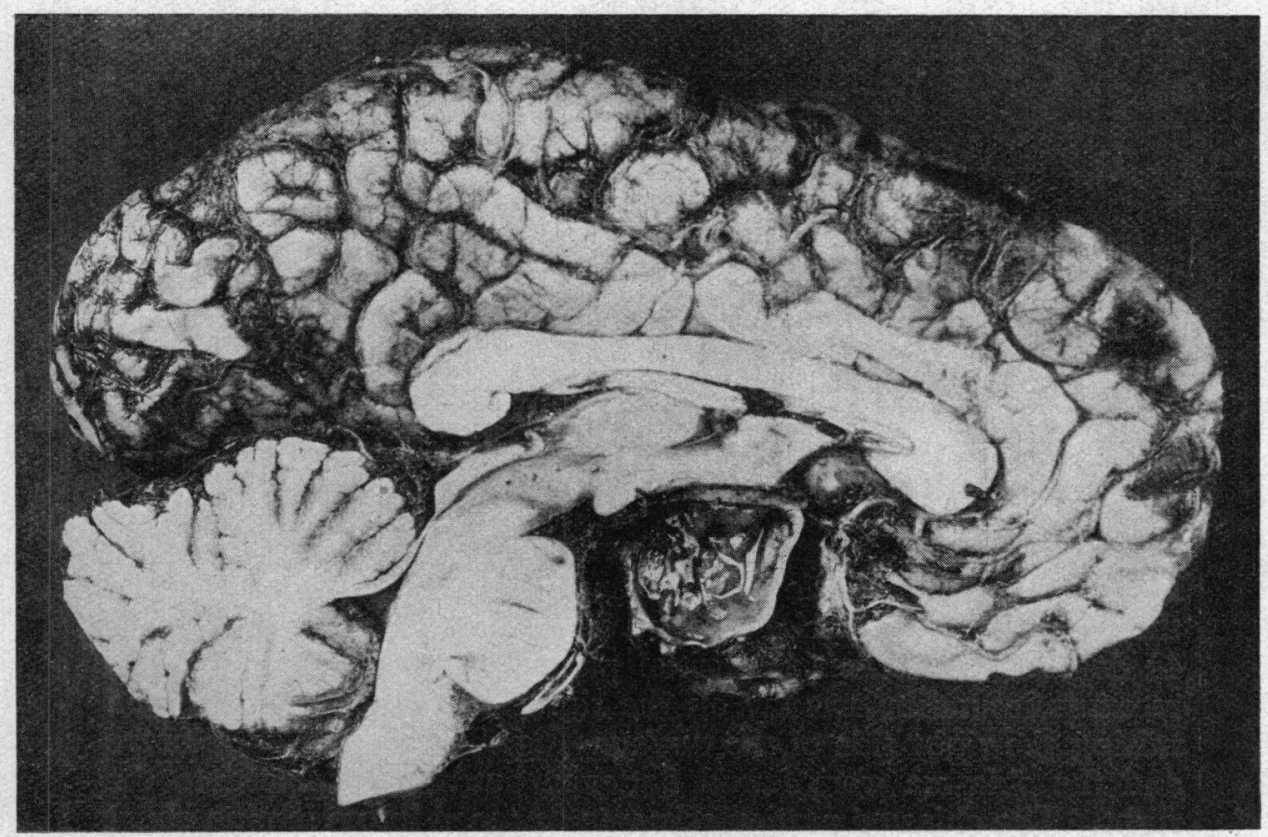

FIG. 2. 
oval opening measuring $1 \mathrm{~mm}$. by $1.5 \mathrm{~mm}$. in diameter by which the tumour communicated with the left anterior cerebral artery (Fig. 2). This artery appeared absolutely normal in every respect, and there was no obvious disease in any of the other arteries of the brain.

The mesial sagittal section cut through the inner border of the left optic thalamus at the middle commissure, as this portion of

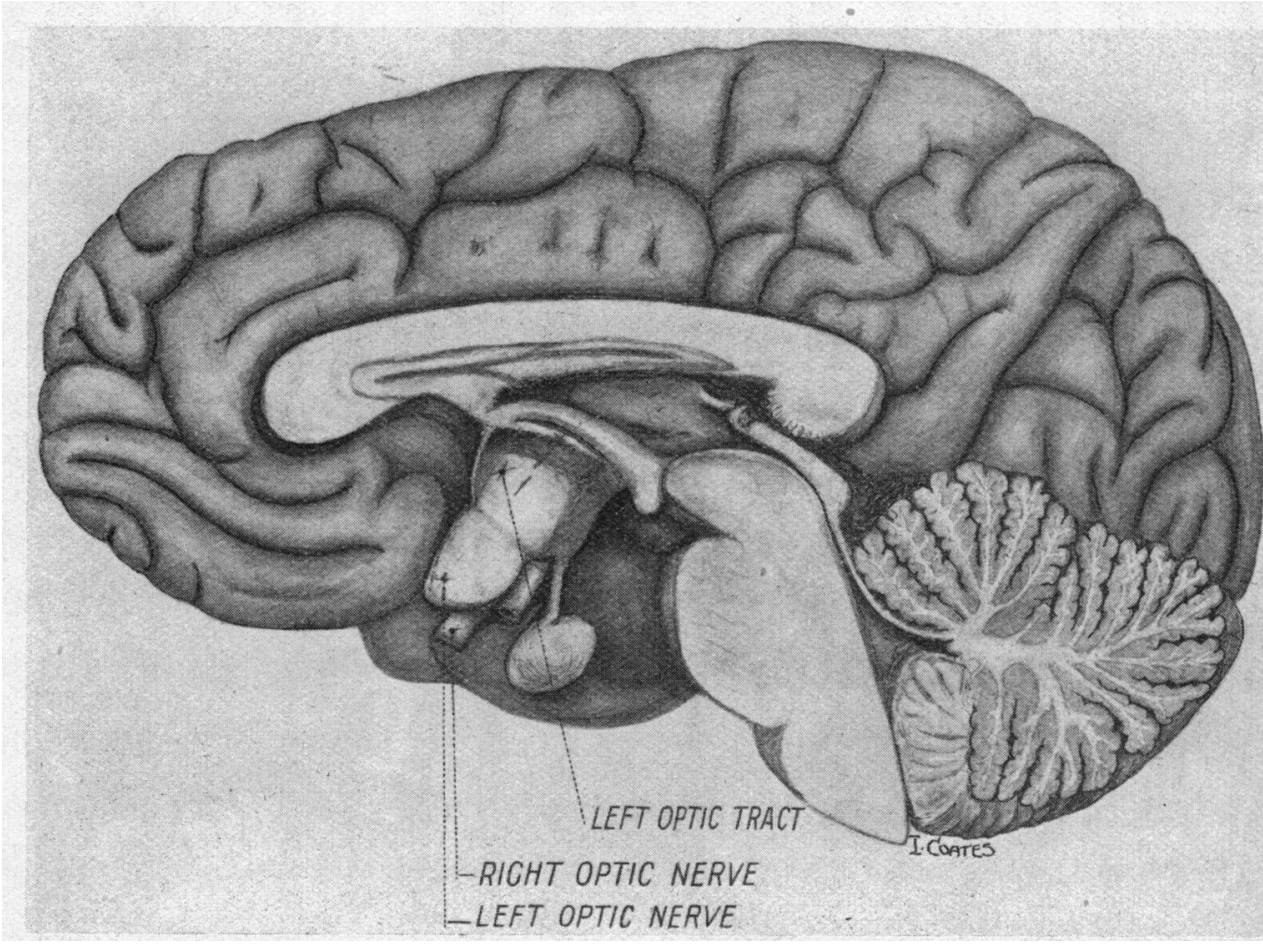

FIG. 3.

the brain was; by pressure, displaced to the right. On the other hand the third ventricle was flattened out and displaced towards the right, so that a probe inserted into it lay at an angle of about 45 degrees to the mesial sagittal plane. As a result of this the recessus infundibularis lay on the right side of the tumour. On raising the right side of the tumour (Fig. 3) the right optic nerve and the chiasma were found flattened out and displaced to the right. The chiasma particularly was flattened into a lamina about two millimetres thick. The right optic tract apparently passed back undisturbed by the tumour, while the left tract was a flattened lamina measuring $12 \mathrm{~mm}$. by $2 \mathrm{~mm}$., which lay on the outer and 
upper aspect of the tumour, compressed between it and the lateral wall of the third ventricle.

From the position of the tumour one might assume that the pressure first affected the left optic tract, then the chiasma, and later the left optic nerve. Each of the oculo-motor nerves appeared on the lateral and inferior aspect of the tumour. The fourth nerves ran more laterally and were apparently not involved by it; the sixth nerves unfortunately could not be dissected out from the dense clot surrounding the tumour after hardening in formalin. The pituitary body was apparently not compressed or otherwise involved. There was no evidence of any serious compression of any other part of the brain.

A microscopical section of the wall of the tumour showed typical vascular tissue with a discontinuous layer of elastic fibres. There were a few small patches of atheroma on its inner aspect.

A few features of this case require discussion. The headache and double vision which characterized the illness of seven years ago were probably due to a small rupture in the aneurysm; the history of many of these cases shows that such sudden attacks occur. They were more commonly due to rupture of the aneurysm, but in some cases the symptoms were probably the result of a sudden swelling of it. In most of the cases headache is a prominent feature, but was.absent in some, as in Freund's.

In our case a radiographic examination revealed no change in the sella Turcica and no shadows. Sosman ${ }^{(8)}$ refers to the fact that in ten out of twenty of the cases of aneurysm at the base of the brain, shadows were seen by X-rays, but only when advanced arterio-sclerotic changes were present. It might be expected from the position occupied by aneurysms that press on the chiasma or optic nerves that ocular palsies would be frequently present, but there is no mention of them in some of the recorded cases. Other neighbouring symptoms might be also expected. In our case no symptoms referable to the pituitary gland were observed, and this has been so in many other cases too. In Kummell's case, however, there was abnormal adiposity, loss of sexual power and other so-called pituitary symptoms.

The variability in visual symptoms which were present in our case might be perhaps regarded as a feature by which the aneurysm could be distinguished from other tumours involving the chiasma and optic nerves. In the cases of Bramwell and Conway a temporary improvement of vision had also occurred. A variability of visual disturbance, however, is not uncommon in pituitary tumours, and Dr. Gordon Holmes has informed me of an unpublished case of endothelioma compressing the chiasma in which vision improved.

Although the pathogenesis of these aneurysms scarcely comes 
into the scope of this paper, it may be briefly remarked that in Freund's case it was most probably syphilitic in origin; in Hutchinson's it was associated with septic endocarditis, and in Bramwell's there was a history of severe cerebral trauma. In my case, however, as in Conway's and others, syphilis could probably be excluded, and there was no arterio-sclerosis or other pathological lesion of the vessels. It is probable that a local congenital weakness of the wall of the artery was the most important factor.

\title{
REFERENCES
}

1. Beadles.-Brain, Vol. XXX, p. 285, 1907.

2. Bramwell.-Edinburgh Med. Jl., Vol. IX, p. 152, 1889.

3. Conway.-Brit. Jl. of Ophthal., Vol. X, p. 78, 1926.

4. Freund.-Klin. Monatsbl. f. Augenheilk., Vol. LVI, p. 468, 1916.

5. Hutchinson.-Trais. Ophthal. Soc., U.K., Vol. IX, p. 152, 1889.

6. Kummell.-Münchener. Med. Wochenschr., Vol. LVIII, p. 1293, 1911.

7. Weir Mitchell. - Jl. of Nervous and Mental Diseases, Vol. XIV, p. 44, 1889.

8. Sosman.-Brit. Jl. of Radiology, Vol. XXX, p. 468, 1925.

9. Stilling.-Zeitschr.f. Ophthal., Vol. III, p. $465,1833$.

\section{A BEND IN THE SIXTH CRANIAL NERVE-AND ITS PROBABLE SIGNIFICANCE}

\author{
E. WolfF \\ LONDON
}

THE weakling of the cranial contents, the sixth nerve may be affected in almost any type of cerebral lesion. It is thus notorious, if involved alone, for having no localizing value.

To quote Uhthoff(1): "Unilateral paralysis of the rectus externus may usually be considered as a pressure or distant symptom in tumour cases especially if incomplete. It often affords no data as to the localization of the process as is shown by the diminution of the paralysis after decompressive operation. It is not justifiable from the unilateral distribution of the paralysis of the external rectus to conclude that the seat of the tumour is on the same hemisphere. I have had cases confirmed by autopsy where the tumour was contralateral."

Many theories have been evolved to account for this.

Collier ${ }^{(2)}$ thought that it was due to a shifting backwards of the brain stem. Those nerves whose direction was most nearly frontocaudal would be involved before the others. Thus the sixth would be first, then the third, and lastly, the seventh and eighth.

But Cushing ${ }^{(3)}$ points out that mere traction or elongation of the nerve does not produce palsy-as is seen especially in cases of cerebello-pontine tumour where the seventh nerve is often stretched to twice its length without resultant facial palsy. 